Check for updates

Cite this: RSC Adv., 2019, 9, 20314

\title{
Platinum on 2-aminoethanethiol functionalized MIL-101 as a catalyst for alkene hydrosilylation $\uparrow$
}

\author{
Zhikai Xie, (D) Weiwen Chen, Xiuying Chen, Xinhua Zhou, Wenbin $\mathrm{Hu}^{*}$ \\ and Xugang Shu*
}

Hydrosilylation is one of the largest-scale applications for homogeneous catalysis and is widely used to enable the commercial manufacture of silicon products. In this paper, a bifunctional heterogeneous catalyst, $\mathrm{Pt}^{\delta+} / \mathrm{AET}-\mathrm{MIL}-101$ (AET $=2$-aminoethanethiol) with a partially positively charged $\mathrm{Pt}^{\delta+}$ electronic structure is reported, which was successfully prepared using post-synthesis modification with AET and a platinum precursor. The catalysts were characterized using X-ray diffraction (XRD), nitrogen $\left(\mathrm{N}_{2}\right)$ adsorption-desorption, transmission electron microscopy (TEM), scanning electron microscopy (SEM) and X-ray photoelectron spectroscopy (XPS) techniques which showed that the synergy of AET-MIL-101 provides a good dispersion of $\mathrm{Pt}^{\delta+}$ in the channels, which can efficiently catalyze the hydrosilylation reaction with almost complete conversion and produce a unique adduct. In addition, the synthetic heterogeneous catalyst $\mathrm{Pt}{ }^{\delta+} / \mathrm{AET}-\mathrm{MIL}-101$ achieves reasonable use of $\mathrm{Pt}$ in terms of number cycles and atomic utilization efficiency, indicating the potential to achieve a green hydrosilylation industry.

Received 24th February 2019

Accepted 16th April 2019

DOI: $10.1039 / c 9 r a 01408 j$

rsc.li/rsc-advances on a styrene-divinylbenzene copolymer (SDB) for the hydrosilylation of various alkenes. The results show that the $\mathrm{Rh}$ is poorly active in the reaction studied, whereas Pt/SDB exhibits excellent catalytic activity. At the same time, by comparing the catalyst carriers: divinylbenzene, activated carbon RX3 extra and carbon black XC72, it showed that the high hydrophobicity and specific surface area of SDB are the focus of the high activity of the catalyst. ${ }^{21}$ The team further compared the effects of bimetallic $\mathrm{Pt}-\mathrm{Cu} / \mathrm{SDB}$ and $\mathrm{Pt} / \mathrm{SDB}$ on the hydrosilylation of allyl chloride, 1-octene and 1-butene. The results show that the bimetallic Pt-copper $(\mathrm{Cu})$ catalyst on the polymer support appears to be much less active than the single metal catalyst. Compared to the Pt/carbon (C) catalyst, SDB seems to be a better carrier material. ${ }^{22}$ In addition, based on the specificity of the structure of silsesquioxane $\left(\mathrm{HSiMe}_{2} \mathrm{O}\right)(\mathrm{i}-\mathrm{Bu})_{7} \mathrm{Si}_{8} \mathrm{O}_{12}$, Walczak et al. decided to examine the hydrosilylation of a wide range of functional alkenes and alkynes (terminal and internal) using $\left(\mathrm{HSiMe}_{2} \mathrm{O}\right)(\mathrm{i}-\mathrm{Bu})_{7} \mathrm{Si}_{8} \mathrm{O}_{12}$, catalyzed by $\mathrm{Pt}$ supported on SDB. The results show that $\mathrm{Pt} / \mathrm{SDB}$ is a more effective catalyst than commercial and industrially applied Pt/C catalysts. The heterogeneous character of the $\mathrm{Pt} / \mathrm{SDB}$ catalyst allows for repeated use up to 20 times and still maintains a high turnover number (TON). Thus, use of the Pt/SDB produced one of the most versatile heterogeneous systems in hydrosilylation reactions. $^{23}$ Chen et al. synthesized a heterogeneous, partially charged single atom $\mathrm{Pt}$ supported on an anatase titanium dioxide $\left(\mathrm{Pt}_{1}{ }^{\delta+} / \mathrm{TiO}_{2}\right)$ catalyst using an electrostatic induction in an exchange two-dimensional confinement strategy. As a result of this they discovering that a partially charged single atom Pt
College of Chemistry and Chemical Engineering, Zhongkai University of Agriculture and Engineering, Guangzhou 510225, China.E-mail:wbhu2000@163.com;xgshu@ 21cn.com; Tel: +8602089003208

$\dagger$ Electronic supplementary information (ESI) available. See DOI: 10.1039/c9ra01408j 
can catalyze the hydrosilylation reaction with almost complete conversion and produce an exclusive adduct. ${ }^{24} \mathrm{Hu}$ et al. reported the synthesis of a super-microporous silica-supported Pt catalyst using $n$-dodecylamine as a surfactant template which was applied for the hydrosilylation of octene with dichloromethylsilane. The catalyst has a conversion of up to $98 \%$ for dichloromethylsilane (99\% selectivity to the $\beta$-adduct). Furthermore, the catalyst can be recycled seven times without significant loss of activity. ${ }^{25}$ However, currently most heterogeneous catalysis still have the disadvantages of low atomic utilization and leaching of active substances. ${ }^{26}$ It is still challenging to develop a heterogeneous catalyst with a combination of the advantages of homogeneous and heterogeneous catalysts.

In recent years, the development of metal-organic frameworks (MOFs) has provided a good carrier for gas storage, ${ }^{27}$ separation, ${ }^{28}$ chemical sensing $^{29}$ and catalysis. ${ }^{30}$ The MOFs are a new type of inorganic-organic porous material coordination polymers. ${ }^{31,32}$ Because MOFs have a larger specific surface area (up to several thousand $\mathrm{m}^{2} \mathrm{~g}^{-1}$ ) and higher volume (up to $90 \%$ of the crystal volume), more controllable pore structure, and so on, ${ }^{33-35}$ the MOFs have been widely used in the field of catalysis. The MOFs have a controlled size distribution and show the tremendous advantage of limiting metal nanoparticles (NPs). When the metal or metal oxide particles are confined in the cavities or the porous channels of MOFs, the prepared catalytic materials had uniform particle dispersion, which is beneficial for the heterogeneous catalyst. ${ }^{36,37}$ However, the absence of functional groups and selective sites in most of the stable MOFs has limited their uses in catalysis. ${ }^{38,39}$ In particular, the MIL-101 type MOF is a one-dimensional porous material formed by an octahedron chrome atom and a terephthalic acid connection (Fig. 1), the interior of which consists of two types of pores [2.9 $\mathrm{nm}$ (a) and $3.4 \mathrm{~nm}$ (b), respectively]. It has a large surface area, multiple unsaturated metal sites and good thermal stability which provides a good site for functionalization. ${ }^{40-42}$ Zhang et al. ${ }^{43}$ reported a highly efficient bifunctional catalyst palladium/sulfonic acid-MIL-101 (chromium) [Pd/SO $\left.\mathrm{SO}_{3} \mathrm{H}-\mathrm{MIL}-101(\mathrm{Cr})\right]$, in which $\mathrm{Pd}$ NPs are uniformly dispersed on a mesoporous $\mathrm{SO}_{3} \mathrm{H}$ functionalized MIL-101(Cr) and exhibit high catalytic performance in the hydrodeoxygenation of vanillin. Xu et al. ${ }^{44}$ successfully encapsulated Pd NPs in cages of ethylenediamine grafted MIL-101 (EDMIL-101), giving highly efficient catalysts (Pd/ED-MIL-101) for

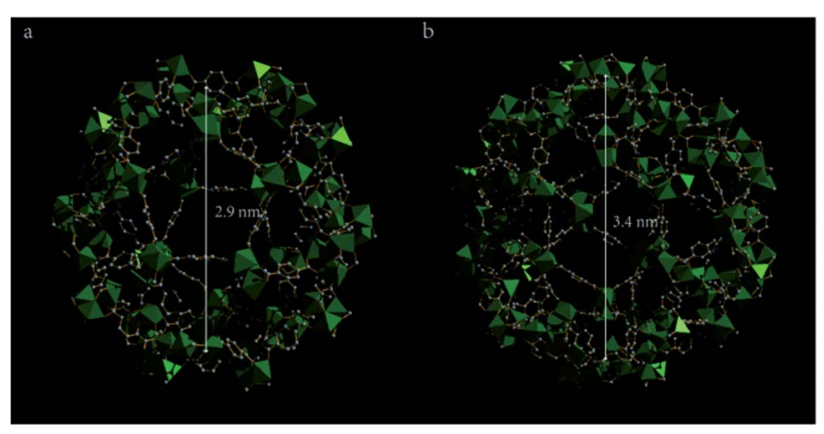

Fig. 1 Ball-and-stick view of MIL-101 showing two types of cages. the racemization of primary amines. Furthermore, the catalyst has excellent stability and can be reused at least eight times without significantly reducing efficiency.

In the present research, 2-aminoethanethiol (AET)-MIL-101 was successfully synthesized using AET functionalized MIL101. Taking advantage of the unique properties of AET-MIL$101, \mathrm{Pt}^{\delta+}$ was uniformly dispersed in the mesoporous cages of AET-MIL-101 to develop a highly efficient catalyst for the catalytic hydrosilylation reaction (Scheme 1). In addition, this paper also explores the commercial value of the catalytic product, and discusses the development prospects for green industrial catalysis.

\section{Experimental}

\subsection{Reagents}

All reagents of analytical or technical grade were purchased and used as received without further purification. Terephthalic acid $\left(\mathrm{H}_{2} \mathrm{BDC}\right)$, chromic nitrate nonahydrate $\left[\mathrm{Cr}\left(\mathrm{NO}_{3}\right)_{3} \cdot 9 \mathrm{H}_{2} \mathrm{O}\right]$, styrene, 1-dodecene, 1-tetradecene and AET were purchased from Macklin Ltd. Hexachloroplatinic acid hydrate $\left(\mathrm{H}_{2} \mathrm{PtCl}_{6}-\right.$ $\left.\cdot 6 \mathrm{H}_{2} \mathrm{O}\right)$ were obtained from Sigma Aldrich Ltd. N,N-Dimethylformamide (DMF), hydrofluoric acid (HF), ethanol, and toluene were purchased from Tianjin Damao Chemical Reagent Factory, Ltd. Heptamethyltrisiloxane (MDHM, MW $=222 \mathrm{~g}$ $\mathrm{mol}^{-1}$ ), acrylic polyether (APEG, MW $=200 \mathrm{~g} \mathrm{~mol}^{-1}$ ) and allyl alcohol polyether $\left(\mathrm{F}-6, \mathrm{MW}=380 \mathrm{~g} \mathrm{~mol}^{-1}\right)$ was kindly provided by Zhejiang Huangma Technology Co., Ltd. Deionized water was obtained from a pure water machine produced by Sichuan Youpu Chaochun Technology Co., Ltd.

\subsection{Preparation of catalyst}

2.2.1 Synthesis of MIL-101. MIL-101 was synthesized according to a method previously described in the literature. ${ }^{36}$ The $\mathrm{Cr}\left(\mathrm{NO}_{3}\right)_{3} \cdot 9 \mathrm{H}_{2} \mathrm{O}(10 \mathrm{mmol}), \mathrm{H}_{2} \mathrm{BDC}(10 \mathrm{mmol})$ and $40 \mathrm{wt} \%$ of $\mathrm{HF}(10 \mathrm{mmol})$ were added to deionized water $(2650 \mathrm{mmol})$. The resulting suspension was stirred for $30 \mathrm{~min}$ at room temperature then heated under autogenous pressure at $220^{\circ} \mathrm{C}$ for $8 \mathrm{~h}$ in a Teflon-lined autoclave. After cooling to room temperature, the green precipitate was collected using centrifugation and washed several times with deionized water and DMF to remove unreacted $\mathrm{H}_{2}$ BDC. The sample was then immersed in ethanol, heated and stirred at $100{ }^{\circ} \mathrm{C}$ for $24 \mathrm{~h}$ for further purification. Finally, the green solid was separated using filtration, and then dried in a vacuum at $80{ }^{\circ} \mathrm{C}$ overnight.

2.2.2 Preparation of AET-MIL-101. The activated MIL-101 $(0.3 \mathrm{~g})$ was dehydrated at $150{ }^{\circ} \mathrm{C}$ in a vacuum oven for $12 \mathrm{~h}$. Then, the dehydrated MIL-101 was dispersed in anhydrous toluene $(30 \mathrm{~mL})$. An appropriate amount of AET $(1.125 \mathrm{mmol})$ was added and the mixture was stirred and refluxed for $12 \mathrm{~h}$ at $110{ }^{\circ} \mathrm{C}$. Finally, the solid product was separated using filtration, washed extensively with excess ethanol and dried at $80{ }^{\circ} \mathrm{C}$ in a vacuum for $12 \mathrm{~h}$. The final product was named AET-MIL-101.

2.2.3 Preparation of $1.5 \% \mathrm{Pt}^{\delta+} /$ AET-MIL-101. First, $0.3 \mathrm{~g}$ of vacuum dried AET-MIL-101 was dispersed in $30 \mathrm{~mL}$ of absolute ethanol, and $0.66 \mathrm{~mL}$ of $\mathrm{H}_{2} \mathrm{PtCl}_{6}$-ethanol $\left(0.035 \mathrm{~mol} \mathrm{~L}^{-1}\right)$ was 


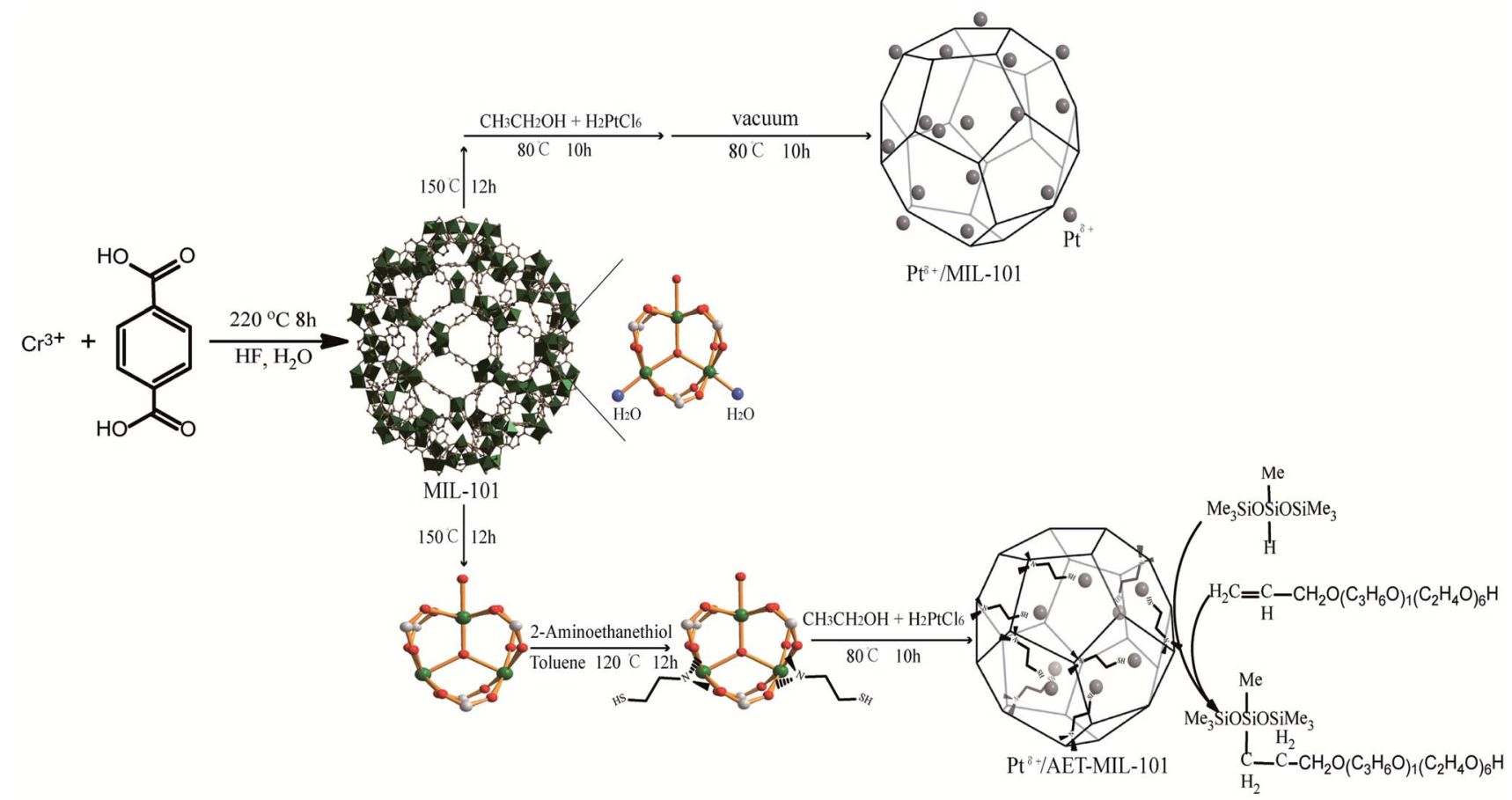

Scheme 1 The strategy for the synthesis of $\mathrm{Pt}^{\delta+} / \mathrm{MIL}-101$ and $\mathrm{Pt}{ }^{\delta+} / \mathrm{AET}-\mathrm{MIL}-101$ catalysts using bi-functionalized MIL-101 and their application in the hydrosilylation process.

added into the suspension. The mixture was mixed ultrasonically for $5 \mathrm{~min}$ in an ultrasonic bath $(100 \mathrm{~W})$ and then stirred vigorously at $80^{\circ} \mathrm{C}$ for $10 \mathrm{~h}$. The solid product was washed with absolute ethanol, and dried at $80{ }^{\circ} \mathrm{C}$ in a vacuum. The asprepared samples were designated as $1.5 \% \mathrm{Pt}^{\delta+} /$ AET-MIL-101, where $1.5 \%$ denotes the nominal Pt loading. For comparison, reference 1.0\% $\mathrm{Pt}^{\delta+} / \mathrm{AET}-\mathrm{MIL}-101,2.0 \% \mathrm{Pt}^{\delta+} / \mathrm{AET}-\mathrm{MIL}-101$ and $1.5 \% \mathrm{Pt}^{\delta+} / \mathrm{MIL}-101$ catalysts were prepared using the same method.

\subsection{Catalyst characterization}

The crystalline structure and phase composition were investigated using X-ray powder diffraction on a D8 ADVANCE (XRD; Bruker) with $\mathrm{Cu} \mathrm{K} \alpha$ radiation operated at $35 \mathrm{kV}$ and $35 \mathrm{~mA}, \lambda=$ $0.154 \mathrm{~nm}$, scanning step $0.02^{\circ}$, scanning range $2-15^{\circ}$. Fourier transform-infrared (FT-IR) spectra were recorded on a Spectrum-100 FT-IR spectrometer (PerkinElmer) using a potassium bromide wafer technique with scanning from 4000 to $450 \mathrm{~cm}^{-1}$. The nitrogen $\left(\mathrm{N}_{2}\right)$ adsorption-desorption isotherms and pore size distribution were obtained at $77 \mathrm{~K}$ using a Quadrasorb SI instrument (Quantachrome Instruments). After the sample was evacuated at $150^{\circ} \mathrm{C}$ for $10 \mathrm{~h}$, the Brunauer-Emmett-Teller (BET) surface area was calculated using the multiple point BET method in the relative pressure range of $P / P_{0}=0.05-0.2$, and the pore diameter distribution curves were obtained using the density functional theory (DFT) calculations method. The morphology of the material and the presence and distribution of Pt was observed using both a S4800 scanning electron microscope (SEM; Hitachi) and a JEM-2100F transmission electron microscopy (TEM; Jeol). The surface electronic states were determined using an ESCALAB 250Xi X-ray photoelectron spectrometer (XPS; ThermoFisher Scientific), and the XPS data were internally calibrated, fixing the binding energy of $\mathrm{C} 1 \mathrm{~s}$ at $284.6 \mathrm{eV}$. The product conversion of various substrates was determined using a $6890 \mathrm{~N}$ gas chromatography-mass spectrometer (GC-MS; Agilent), equipped with an HP-5MS capillary column $(30 \mathrm{~m} \times 0.25 \mathrm{~mm}, 0.25 \mu \mathrm{m})$. An auto-injector was used to inject the samples at $250^{\circ} \mathrm{C}$ and was set to a $10: 1$ split ratio. The carrier gas flow rate of helium was $1 \mathrm{~mL} \min ^{-1}$. The warming procedure was as follows: held at $50{ }^{\circ} \mathrm{C}$ for $2 \mathrm{~min}$, ramped to $280{ }^{\circ} \mathrm{C}$ at a rate of $15{ }^{\circ} \mathrm{C} \mathrm{min}^{-1}$ and then held for $10 \mathrm{~min}$. The ionization mode was electron ionisation $(70 \mathrm{eV}, 300$ $\mu \mathrm{A})$ and the ion source of the MS was set to $230^{\circ} \mathrm{C}$. Proton nuclear magnetic spectrometry $\left({ }^{1} \mathrm{H}\right.$-NMR) spectra were recorded on an Ascend 400 (400 MHz, Bruker) NMR spectrometer using deuterated chloroform as solvent. The Pt content of the catalyst was determined using an iCAP 6000 Series inductively coupled plasma emission spectrometer (ICP; ThermoScientific). The water or diluted catalytic product $\left(V_{\text {product }}: V_{\text {water }}=1: 1000\right)$ droplet contact angles and surface tension were measured at room temperature on a self-supporting pressed sample disc using a Attention Theta contact angle measuring system (Biolin Scientific). By slowly dripping the liquid on to the surface of cucumber leaves, the contact angle was determined from images captured within $7 \mathrm{~s}$ using a high-performance coupled device camera.

\subsection{Catalytic performance evaluation}

Typical hydrosilylation reaction procedures were as follows. A mixture of the catalyst $\left(\mathrm{Pt}^{\delta+} / \mathrm{AET}-\mathrm{MIL}-101\right.$ or $\left.\mathrm{Pt}^{\delta+} / \mathrm{MIL}-101\right)$ and 
varying amounts of F-6 were added into $50 \mathrm{~mL}$ three-necked flasks vigorously stirred at $80{ }^{\circ} \mathrm{C}$ for $30 \mathrm{~min}$. Then, $0.035 \mathrm{~mol}$ of MDHM was added dropwise under stirring and the reaction mixture was heated up to a certain temperatures. After cooling to room temperature, the product was separated using centrifugation $\left(6000 \mathrm{rpm}, 20 \mathrm{~min}, 25^{\circ} \mathrm{C}\right)$ and further characterized using GC-MS and ${ }^{1} \mathrm{H}$-NMR (see S2 and S3, ESI $\dagger$ ). Finally, catalytic recycling was studied using the recovered catalysts.

\section{Results and discussion}

\subsection{Catalyst characterization}

The powder XRD pattern of the synthesized MIL-101 (Fig. 2) matched well with the powder XRD pattern diffraction peak of MIL-101 in the single crystal data simulation pattern, and there was no apparent loss, the main characteristic diffraction peaks appeared at $2 \theta=2.96^{\circ} .3 .44^{\circ}, 4.46^{\circ}, 5.28^{\circ}, 6.01^{\circ}, 8.55^{\circ}$, and $9.18^{\circ}$, which indicated good phase purity of the synthesized MIL-101 carrier. After the introduction of AET on the backbone of MIL-101 and the incorporation of Pt, no significant change in peak position was observed, indicating that the crystal structure of MIL-101 was well maintained during grafting and doping. Additionally, the peak intensity at $3.44^{\circ}$ dramatically decreased, which was because the MIL-101 channels were partially filled with AET and Pt. ${ }^{45}$ The FT-IR spectra of MIL-101 and AET-MIL101 are shown in Fig. 3. Comparing the two spectra, it can be observed that two new characteristic peaks appeared in MIL-101 modified with AET. Among them, weak absorption peak appeared at $2978 \mathrm{~cm}^{-1}$, which belong to the $-\mathrm{CH}_{2}-\mathrm{CH}_{2}-$ stretching vibration, whereas the sharp peak at about $1043 \mathrm{~cm}^{-1}$ indicated the stretching vibration of the $\mathrm{C}-\mathrm{N}$ bond. However, no characteristic stretching vibration peak of the $-\mathrm{NH}_{2}$ group was observed, possibly because of the combination with $\mathrm{Cr}^{3+}$ being shielded.

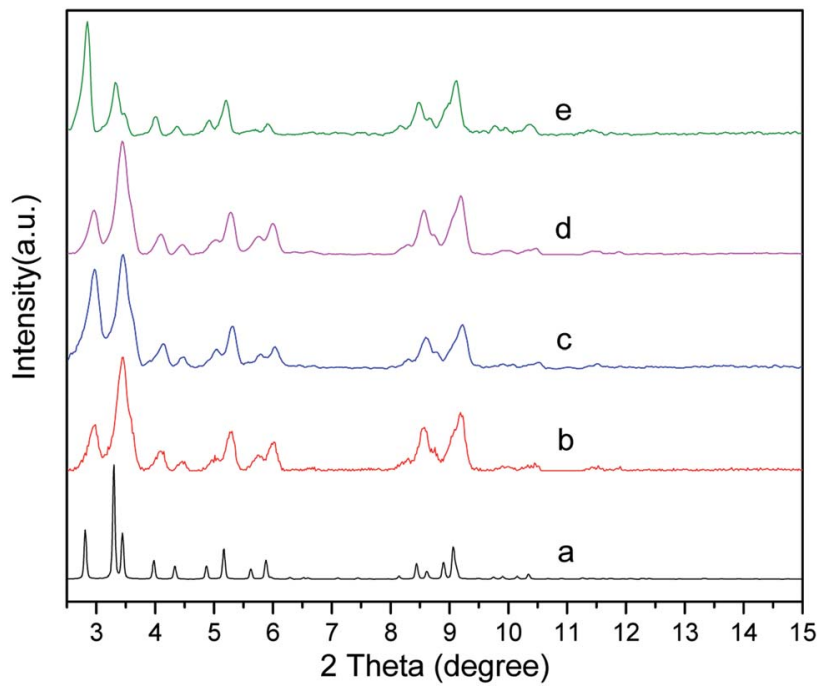

Fig. 2 XRD patterns of MIL-101 simulated from crystal structure data (a), MIL-101 (b), 1.5\% $\mathrm{Pt}^{\delta+} / \mathrm{MIL}-101$ (c), AET-MIL-101 (d) and $1.5 \% \mathrm{Pt}^{\delta+} /$ AET-MIL-101 (e).

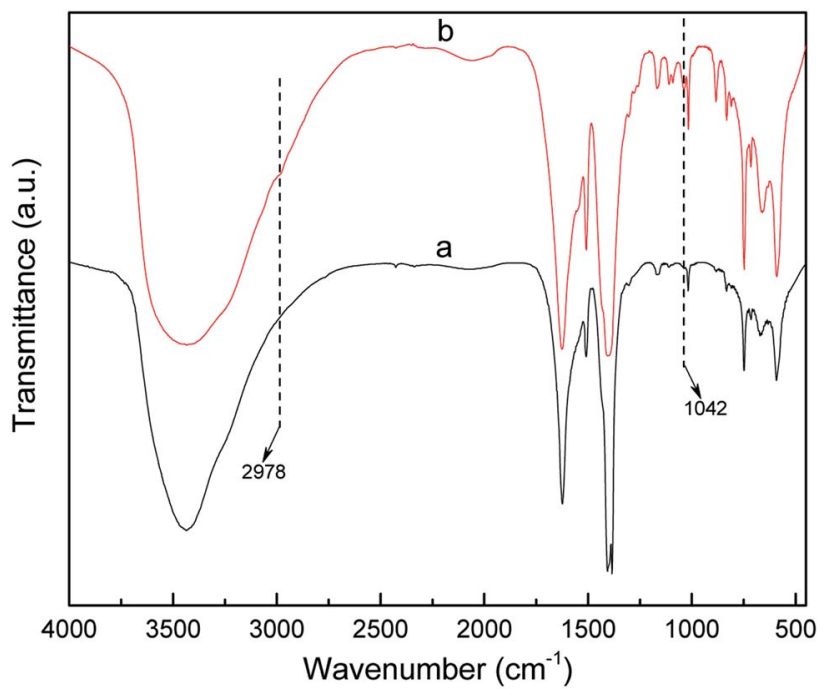

Fig. 3 FT-IR spectra of MIL-101 (a) and AET-MIL-101 (b).

The surface morphology of unmodified and modified MIL101 samples was characterized using SEM and TEM (Fig. 4). The MIL-101 mainly exhibited uniformly sized octahedron
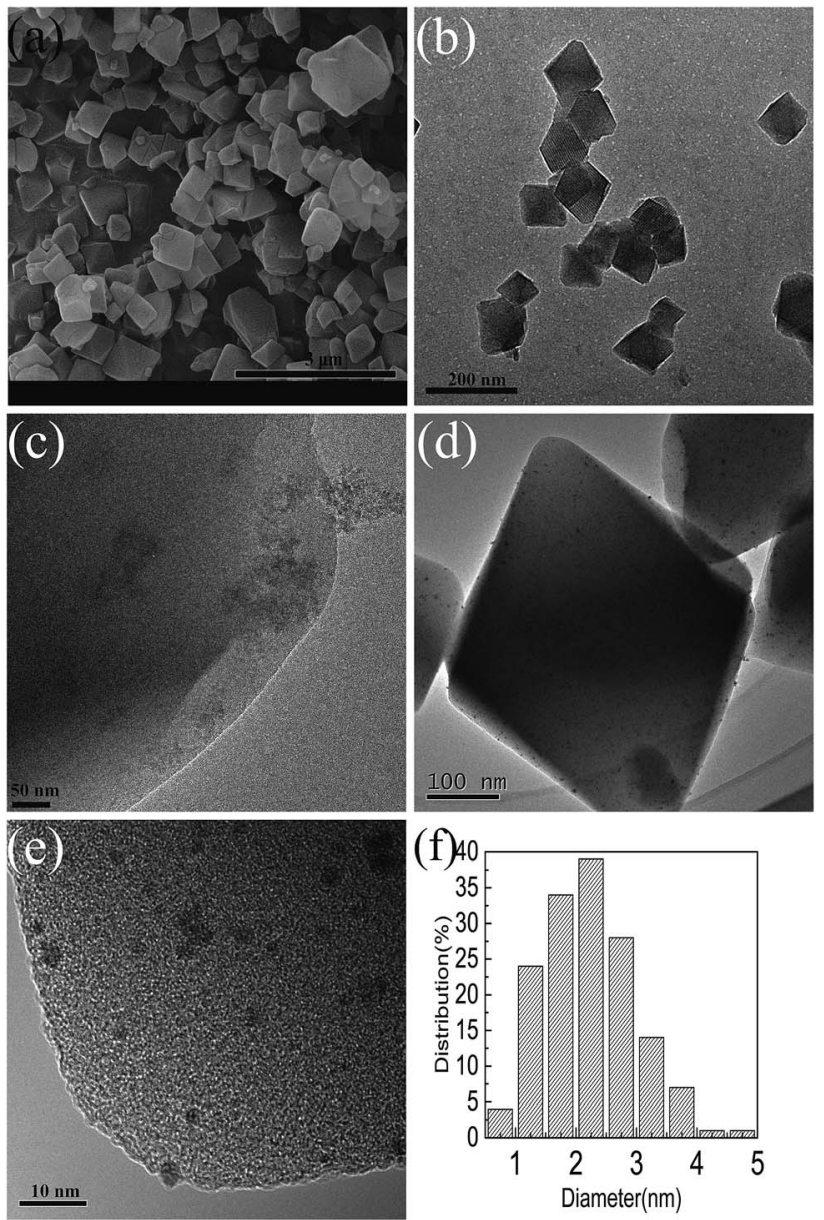

Fig. 4 SEM images of MIL-101 (a), TEM images of MIL-101 (b), AETMIL-101 (c), 1.5\% Pt ${ }^{\delta+} / \mathrm{AET}-\mathrm{MIL}-101$ (e and d) and the corresponding size distribution plot of $\mathrm{Pt}^{\delta+}(\mathrm{f})$. 
morphology (Fig. 4a) and a good lattice structure (Fig. 4b), indicating that the material had good crystallinity. The MIL-101 modified by AET clearly shows a small amount of aggregate formation (Fig. 4c), which could be because of the formation of aggregates of AET crystals. After Pt was incorporated into the carrier AET-MIL-101, only a few $\mathrm{Pt}^{\delta+}$ appear on the outside surface of the parent carrier (Fig. 4d), whereas most of the metal $\mathrm{Pt}^{\delta+}$ was confined to the cages of the carrier (Fig. 4e). This demonstrated that the $\mathrm{Pt}^{\delta+}$ was well dispersed in the AET-MIL101 guest molecule, had a narrow size distribution between $1 \mathrm{~nm}$ and $4 \mathrm{~nm}$ (Fig. 4f), and was concentrated around $2 \mathrm{~nm}$. This may be attributed to the stability of the structure of the crystal itself and the presence of functional groups attached to the framework.

The $\mathrm{N}_{2}$ adsorption-desorption isotherms and the pore size distribution profiles are shown in Fig. 5 . The BET surface area and pore volume of MIL-101 loaded with $\mathrm{Pt}^{\delta+}$ did not change significantly (Table 1), and this was mainly because $\mathrm{Pt}^{\delta+}$ were well dispersed on the support or most of the $\mathrm{Pt}^{\delta+}$ was concentrated on the outer surface. After modification with AET, the $\mathrm{N}_{2}$ adsorption of the AET-MIL-101 samples decreased significantly, and the corresponding BET surface area was reduced to $1603 \mathrm{~m}^{2}$ $\mathrm{g}^{-1}$. The pore volume was reduced to $0.756 \mathrm{~cm}^{3} \mathrm{~g}^{-1}$, and the average pore diameter also decreased rapidly. However, the pore sizes and pore volume of AET-MIL-101 loaded with $\mathrm{Pt}^{\delta+}$ were also reduced compared to those of the AET-MIL-101 samples, which were $1454 \mathrm{~m}^{2} \mathrm{~g}^{-1}$ and $0.661 \mathrm{~cm}^{3} \mathrm{~g}^{-1}$, respectively, indicating that most of the AET and $\mathrm{Pt}^{\delta+}$ were dispersed inside the pores instead of being distributed on the outer surface.

Fig. 6A shows the XPS survey data and indicates that $1.5 \%$ $\mathrm{Pt}^{\delta+} /$ AET-MIL-101 contained six elements C, Cr, N, O, Pt, and, S indicating that AET was successfully modified to MIL-101. The S 2p spectrum of the catalyst showed two different peaks (Fig. 6C),
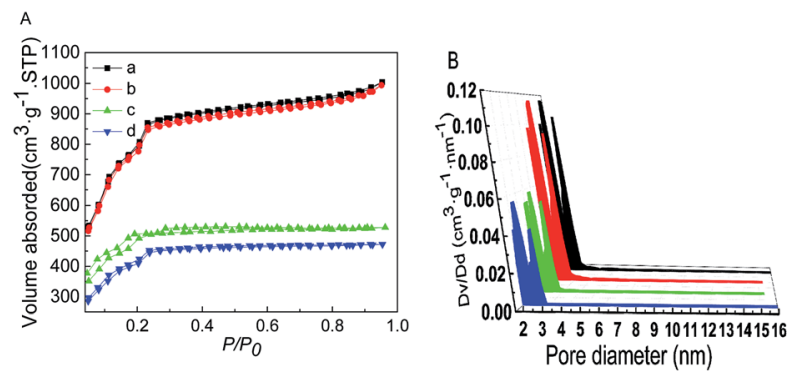

Fig. 5 The $\mathrm{N}_{2}$ isotherms at $-196^{\circ} \mathrm{C}$ (A) and DFT pore size distributions (B) of MIL-101 (a), 1.5\% $\mathrm{Pt}^{\delta+} / \mathrm{MIL}-101$ (b), AET-MIL-101 (c) and 1.5\% $\mathrm{Pt}^{\delta+} /$ AET-MIL-101 (d).

Table 1 Selected structural properties of the catalysts

\begin{tabular}{lll}
\hline Sample & & $\begin{array}{l}\text { Pore volume } \\
\left(\mathrm{cm}^{3} \mathrm{~g}^{-1}\right)\end{array}$ \\
\hline MIL-101 & 2877 & 1.39 \\
$1.5 \% \mathrm{Pt}^{\delta+} / \mathrm{MIL}-101$ & 2823 & 1.36 \\
$\mathrm{AET}-\mathrm{MIL}^{2}-101$ & 1603 & 0.76 \\
$1.5 \% \mathrm{Pt}^{\delta+} /$ AET-MIL-101 & 1454 & 0.66
\end{tabular}

which can be attributed to the $\mathrm{S}-\mathrm{H}(163.7 \mathrm{eV})$ and $\mathrm{Pt}-\mathrm{S}$ bonds $(164.1 \mathrm{eV})$. The oxidation state of $\mathrm{Pt}$ in $\mathrm{Pt}^{\delta+} /$ AET-MIL-101 was determined using the data in Fig. 6D. The Pt $4 \mathrm{f}$ spectrum could be deconvolved into two peaks at binding energies of $76.0 \mathrm{eV}$ and $72.8 \mathrm{eV}$, corresponding to the $4 \mathrm{f}_{5 / 2}$ and $4 \mathrm{f}_{7 / 2}$ levels, respectively. The peak positions are between $\mathrm{Pt}(\mathrm{II})$ and $\mathrm{Pt}(0)$, which indicated that the Pt atom carried a portion of the positive charge from the electron transfers between the metal and the support because of the enhanced metal-support interaction. ${ }^{24}$ In terms of kinetics, because the partially charged $\mathrm{Pt}^{\delta+}$ electronic structure of $\mathrm{Pt}^{\delta+} /$ AET-MIL-101 was produced before the catalytic reaction proceeded, it did not need to undergo additional formation to initiate the hydrosilylation reaction compared to the other forms. Thus, the $\mathrm{Pt}^{\delta+} /$ AET-MIL-101 catalyst has a higher catalytic performance for the hydrosilylation of alkenes. ${ }^{46}$

\subsection{Catalytic hydrosilylation of alkenes}

A number of related reports have demonstrated that Pt was an efficient catalyst for the hydrosilylation of alkenes. However, because of its environmentally friendly nature and ecological protection, the above catalyst was applied to catalyze the hydrosilylation of F-6 as a representative substrate for MDHM. There was no need to add other organic solvents or additives, and the product is environmentally friendly and non-toxic, with excellent wettability, ductility and permeability. Therefore, it has a certain use in industrial production. ${ }^{15}$

The effects of different loadings of $\mathrm{Pt}^{\delta+} / \mathrm{AET}-\mathrm{MIL}-101$ and separate factors on the catalytic activity of F-6 and MDHM hydrosilylation reactions were investigated. The catalytic results are summarized in Table 2. Under the same catalytic conditions, the catalytic effect of the $1.5 \% \mathrm{Pt}^{\delta+} / \mathrm{AET}-\mathrm{MIL}-101$ catalyst was better than that of the $1 \%$ and $2 \%$ catalysts (Table 2 , entries $6,13,14)$. However, using the pure AET-MIL-101 support or MIL-101, virtually no reaction took place (only about 3\% conversion), implying that the use of Pt is inevitable for catalyzing alkene hydrosilylation (Table 2, entries 1 and 2).
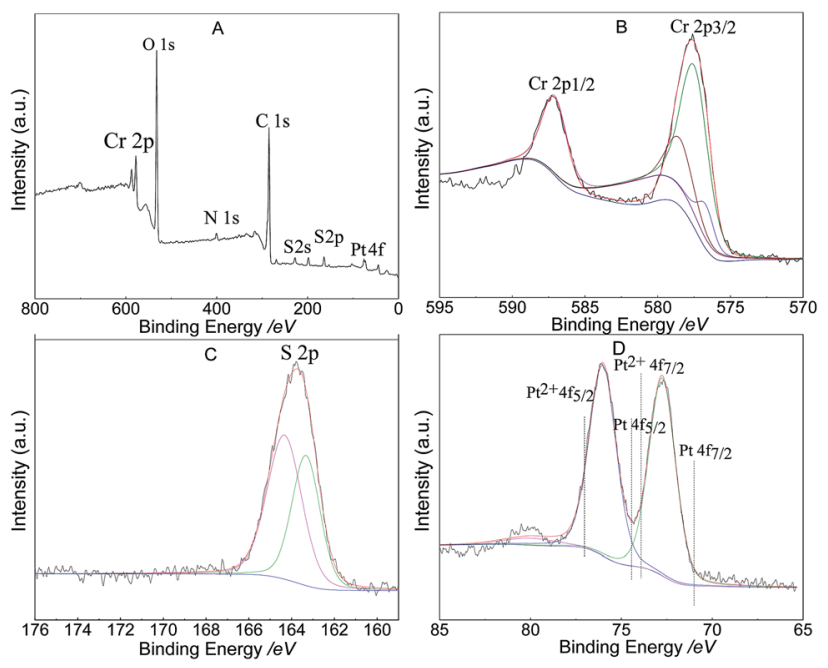

Fig. 6 XPS spectra of 1.5\% $\mathrm{Pt}^{\delta+} / \mathrm{AET}-\mathrm{MIL}-101$ : survey spectrum (A), high resolution $\mathrm{Cr}$ spectrum (B), high resolution $\mathrm{S}$ spectrum (C) and high resolution Pt spectrum (D). 
Table 2 The effect of various factors on the hydrosilylation conversion of F-6

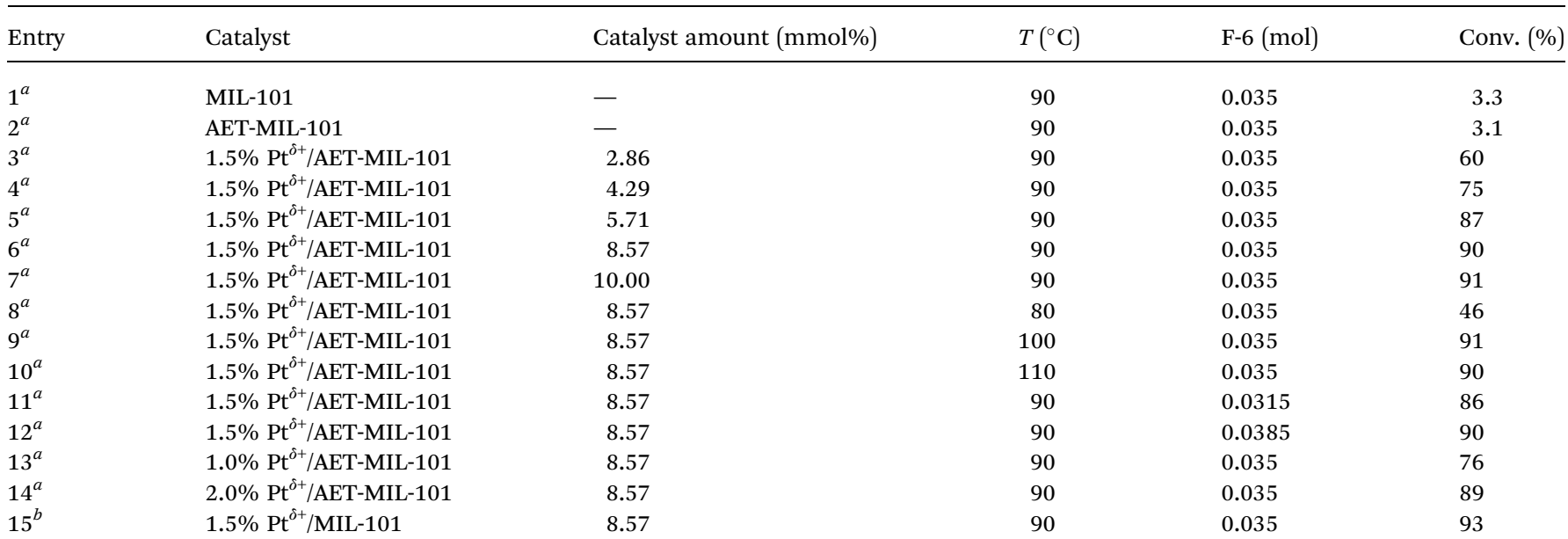

${ }^{a}$ Reaction conditions: $\mathrm{MDHM}=0.035 \mathrm{~mol}$, reaction time: $180 \mathrm{~min}$, feeding method: MDHM is added dropwise to alkenes. ${ }^{b}$ Reaction conditions: $\mathrm{MDHM}=0.035 \mathrm{~mol}$, reaction time: $30 \mathrm{~min}$, feeding method: MDHM is added dropwise to alkenes.

The hydrosilylation reaction was carried out at $90{ }^{\circ} \mathrm{C}$ with different low doses (relative to the amount of Pt in MDHM) of $\mathrm{Pt}^{\delta+} / \mathrm{AET}-\mathrm{MIL}-101$ (1.5\% Pt loading) catalyst. It was found that as the dose increased, the conversion rate of the substrate also showed an upward trend, and the dosages of $8.57 \mathrm{mmol} \%$ and $10.00 \mathrm{mmol} \%$ showed better conversion (Table 2, entries 3-7). This was because the unit catalyst and the unit substrate used for the catalytic reaction at a dosage of $8.57 \mathrm{mmol} \%$ forms a saturated state. Thus, further research was conducted with a catalyst amount of $8.57 \mathrm{mmol} \%$. The reaction temperature acts as an important factor affecting the activity of the catalyst. The reaction at $80{ }^{\circ} \mathrm{C}$ for $180 \mathrm{~min}$ only had a conversion of $46 \%$, and further increasing the reaction temperature led to a remarkable increase in the reaction rate (Table 2, entries 6 , 8-10), which could reach $90 \%$ when the temperature was raised to $90{ }^{\circ} \mathrm{C}$.

The conversion of MDMA was further investigated by sampling every $45 \mathrm{~min}$ at each temperature (Fig. 7). The reaction was accompanied by a time increase, each curve showed a tendency to rise first and then stabilize. As shown in Fig. 7, the MDHM conversion at $90{ }^{\circ} \mathrm{C}$ was significantly higher than $100{ }^{\circ} \mathrm{C}$ and $110{ }^{\circ} \mathrm{C}$ in a relatively short period of time. This may be because the temperature was too high causing some of the MDHM to be vaporized and reflux rereacted. This is due to the excessive temperature causing a portion of the MDHM to be refluxed into the flask to participate in the reaction again. As the reaction progressed, most of the MDHM participates in the reaction, and the reflux rate gradually decreases. There was almost no reflux of MDHM after $180 \mathrm{~min}$. Then the conversion of the reaction was compared by adding different molar ratios of the substrates. Different molar ratios of the substrates show good catalytic efficiency (Table 2, entries 6, 11 and 12). According to the mechanism of the hydrosilylation reaction, the optimum conversion could be achieved when the molar ratio was increased to $1: 1$ (90\% conversion).
Finally, the catalytic activities of the different catalysts $\mathrm{Pt}^{\delta+} /$ MIL-101 were compared (Table 2, entries 6 and 15), ${ }^{47}$ although the $\mathrm{Pt}^{\delta+} / \mathrm{MIL}-101$ catalyst has better catalytic activity (conversion: 93\%). However, in the experiment, it was found that its catalytic rate was too fast during the reaction, because of a sharp rise in temperature, and difficulty in controlling the reaction rate. This was a reaction phenomenon similar to homogeneous catalysis, which is unfavorable for the protection of industrial equipment.

In general, according to the Chalk-Harrod theory, ${ }^{48}$ hydrosilylation can proceed by the following four steps: (i) activation by Pt, (ii) hydrosilane addition, (iii) alkenes are inserted into the $\mathrm{Pt}-\mathrm{H}$ bond, and (iv) product shedding. The activation priority of hydrosilylation was studied by changing the addition order of

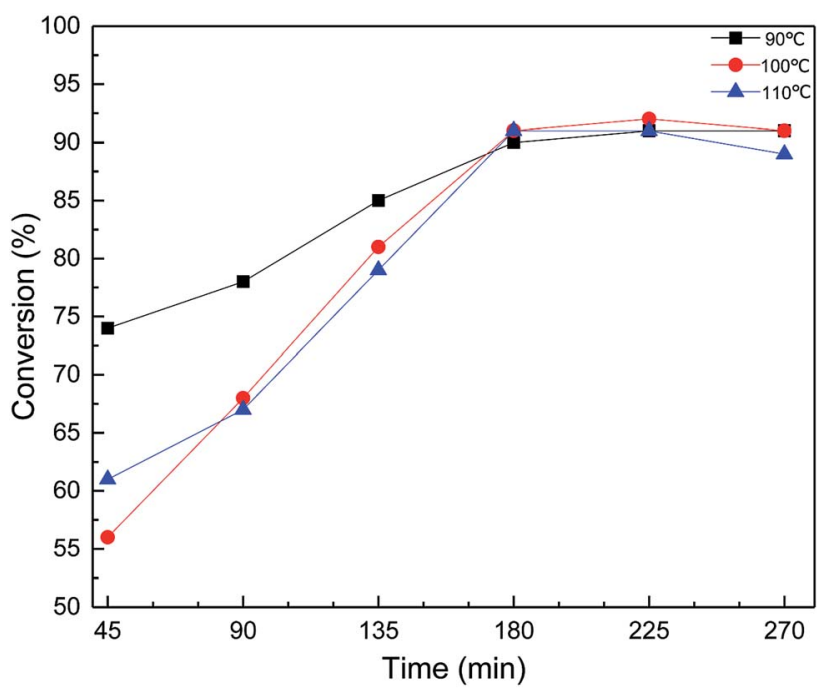

Fig. 7 Effect of $\mathrm{Pt}^{\delta+} / \mathrm{AET}-\mathrm{MIL}-101$ on MDHM conversion at different temperatures. Reaction conditions: MDHM: 0.035 mol, F-6: $0.035 \mathrm{~mol}$, amount of catalyst: $39 \mathrm{mg}$, feeding method: MDHM is added dropwise to the alkenes. 
the substrates. The results show that although the three substrate addition methods can achieve excellent conversion, the conversion rate of different addition methods follows this trend: $\mathrm{a}>\mathrm{c}>\mathrm{b}$. Thus, it is believed that the activation before the reaction is beneficial to the smooth progress of step (i). It can increase the reaction rate and reduce the reaction time. According to the a and c curves in Fig. 8, it can be seen that both reactants can be activated by $\mathrm{Pt}^{\delta+}$, but under the same reaction conditions, the alkene will participate in step (i) earlier than MDHM.

The reusability and stability of the heterogeneous catalysts was of significant importance in terms of industrial applications and green chemistry. To address the concerns, the catalyst can easily be separated from the reaction solution by a simple centrifugal separation operation, followed by washing it several times with ethanol and then using it for cyclic experiments after vacuum drying. In a series of consecutive runs, the catalyst was found to be highly stable and catalytic activity could still be maintained at about $84 \%$ after repeated use for five cycles as shown in Fig. 9. In addition, the recovered catalyst was characterized using XRD, ICP and XPS analysis. The XRD analysis of the recovered catalyst (S1(b), ESI $\dagger$ ) showed that its crystallinity and the crystallinity of the fresh catalyst remained substantially unchanged, and the loading of Pt was reduced to $1.18 \%$ from the original $1.47 \%$. The XPS of the recovered catalyst showed that the catalyst retained the $\mathrm{Pt}^{\delta+}$ morphology (S2, ESI $\dagger$ ) after several cycles. Finally, in order to explore the versatility of this catalyst, the hydrosilylation reactions were carried out under the previously mentioned optimal conditions using various alkenes (Table 3 ). Although the conversion changed as the molecular chain grew, the catalytic effect was satisfactory.

\subsection{Performance test of hydrosilylation products}

The contact angles of water and the catalytic products on cucumber leaves are shown in Fig. 10. Because the cucumber

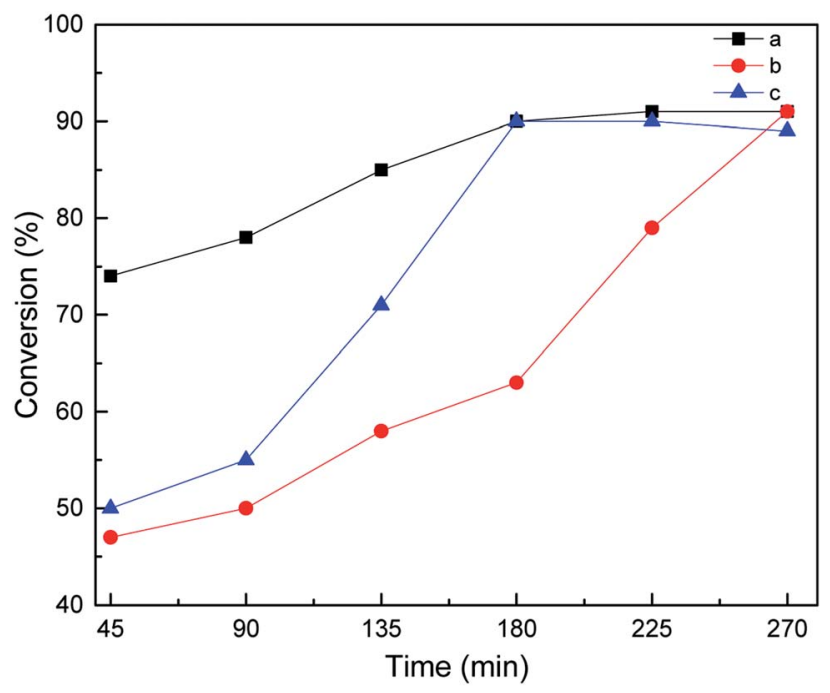

Fig. 8 Effect of feeding mode on MDHM conversion, feeding method: MDHM is added dropwise to alkenes (a), mixed alkenes are added together with MDHM (b), alkenes are added dropwise to MDHM (c).

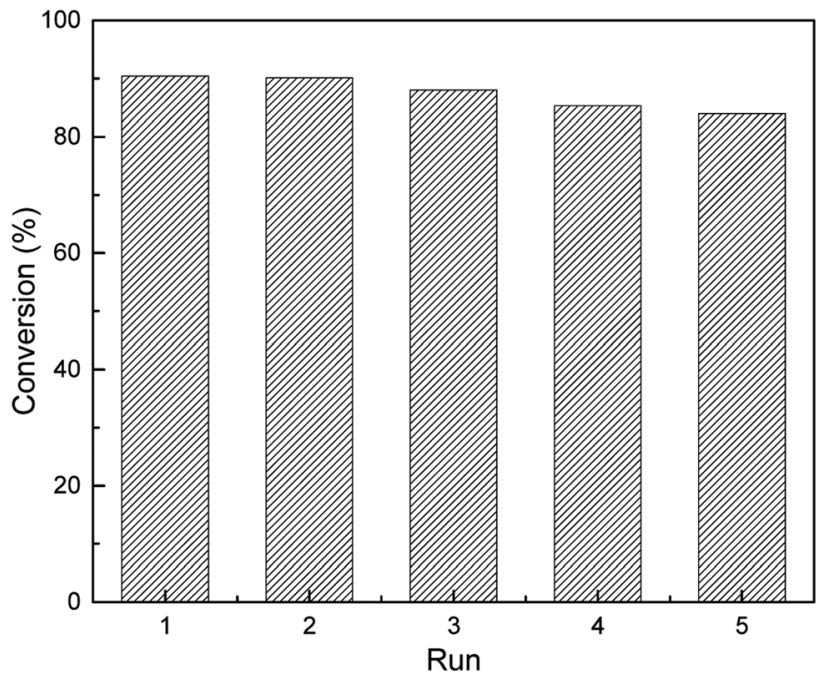

Fig. 9 Reusability of $1.5 \% \mathrm{Pt}^{\delta+} / \mathrm{AET}-\mathrm{MIL}-101$ in the catalytic hydrosilylation of F-6. Reaction conditions: MDHM: $0.035 \mathrm{~mol}$, F-6: $0.035 \mathrm{~mol}$, amount of catalyst: $39 \mathrm{mg}$, reaction temperature: $90{ }^{\circ} \mathrm{C}$, reaction time: $180 \mathrm{~min}$, feeding method: MDHM is added dropwise to alkenes.

itself is highly hydrophobic, the angles observed remain unchanged after adding water droplets to the cucumber leaves for $7 \mathrm{~s}$ (Fig. 10a-c). However, by adding a small amount of the catalytic product, the contact area of water on the cucumber leaf surface can be greatly increased, and only $0.58 \mathrm{~s}$ was required to completely spread the water on the leaf surface (Fig. 10d-f). This was because of the surface properties of the catalytic product are imparted by the crosslinking of the functional silanes with the polymer, and thus, good wettability was obtained. Therefore, the product shows good prospects for future applications in agriculture and fine chemicals.

At the same time, the same treatment method was used to test the surface tension of the product. The test results are shown in Table 4 . The hydrosilylation product synthesized from $\mathrm{Pt}^{\delta+} /$ AET-MIL-101 had an average surface tension of $19.32 \mathrm{mN} \mathrm{m}^{-1}$ and the surface tension of water was 67.77 $\mathrm{mN} \mathrm{m}^{-1}$, indicating that the product synthesized by the catalyst could significantly reduce the surface tension of water.

Table 3 Hydrosilylation of various alkenes and MDHM using the $\mathrm{Pt}^{\delta+}$ / AET-MIL-101 catalyst $^{a}$

\begin{tabular}{llll}
\hline Entry & Substrate & Conv. $(\%)$ & TOF $\left(\mathrm{h}^{-1}\right)$ \\
\hline 1 & Ally alcohol polyether (380) & 90 & $8.3 \times 10^{3}$ \\
2 & Acrylic polyethers (200) & 90 & $7.6 \times 10^{3}$ \\
3 & 1-Dodecene & 99 & $1.1 \times 10^{4}$ \\
4 & 1-Tetradecene & 95 & $1.0 \times 10^{4}$ \\
5 & Styrene & 89 & $5.0 \times 10^{3}$
\end{tabular}

${ }^{a}$ Reaction conditions: amount of catalyst: $39 \mathrm{mg}$ (Pt $\left.8.57 \mathrm{mmol} \%\right)$, MDHM: $0.035 \mathrm{~mol}$, reaction time: $180 \mathrm{~min}$, temperature: $90{ }^{\circ} \mathrm{C}$, MDHM : alkene molar ratio: $1: 1$, feeding method: MDHM is added dropwise to alkene. Data in parentheses represent the molecular weight of the alkene. 


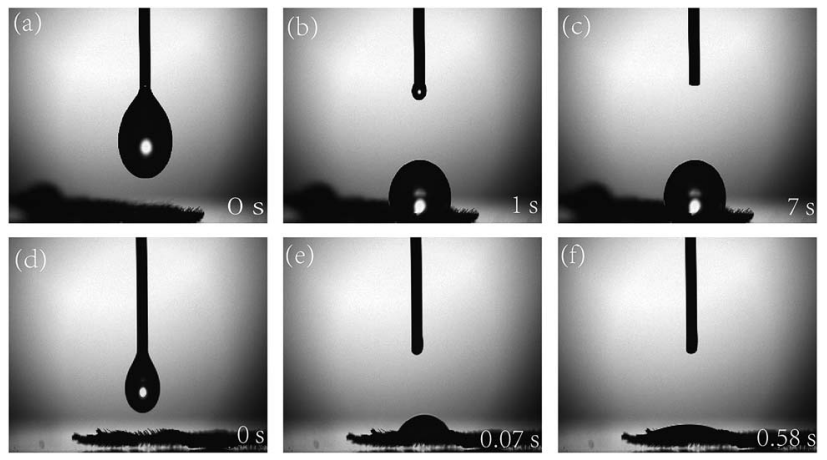

Fig. 10 Contact angles of water droplets on cucumber leaves (a), stationary $1 \mathrm{~s}$ (b), stationary $7 \mathrm{~s}$ (c), adding a small amount of the catalytic products to water (d), stationary $0.07 \mathrm{~s}(\mathrm{e})$ and stationary 0.58 $s(f)$.

Table 4 Comparison of the surface tension of various products in water

\begin{tabular}{lccc}
\hline Substance & \multicolumn{3}{c}{ Surface tension $\left(\mathrm{mN} \mathrm{m}^{-1}\right)$} \\
\hline No added catalytic product & 68.05 & 67.58 & 68.68 \\
Catalytic product added & 19.26 & 19.33 & 19.37 \\
Homogeneous catalytic product & 19.29 & 19.34 & 19.33 \\
added & & & \\
\end{tabular}

\section{Conclusion}

In summary, a new and robust heterogeneous catalyst was developed by encapsulating Pt into MIL-101 cages modified with AET. Pt as highly catalytically active sites for AET-MIL-101 for the alkenes hydrosilylation under mild reaction conditions and exhibits excellent reusability. The unexpected property of $\mathrm{Pt}^{\delta+} /$ AET-MIL-101 can be attributed to unique partially positive valence electronic structure and the highly dispersible properties of the Pt species. The stable performance of $\mathrm{Pt}^{\delta+} / \mathrm{AET}$-MIL101 achieves a reasonable use of the catalysts by reducing consumption of the precious platinum by increased recycling and greatly increases the atomic utilization efficiency in the form of highly dispersed $\mathrm{Pt}^{\delta+}$. Meanwhile, the findings highlight the fact that the addition of modifiers on the surface of the cages in MIL-101 give the material better stability and controllability of the catalytic rate compared to the unmodified MIL101. In addition, it was shown through this research that the catalytic products have excellent interfacial properties, which will help to achieve the green hydrosilylation for agricultural and industrial applications.

\section{Conflicts of interest}

There are no conflicts to declare.

\section{Acknowledgements}

The project was supported by the National Natural Science Foundation of China (No. 21476272), Provincial Department of Agriculture Project of Guangdong (KB1814324, KB1814325),
Science and Technology Planning Project of Guangdong Province of China (2017A050506055) and the Graduate Education Innovation Program (KA180582712).

\section{References}

1 J. S. Rhee, R. M. Sneeringer and A. S. Penzias, Coord. Chem. Rev., 2011, 255, 1440-1459.

2 H. Wu, C. Zheng, N. Chen, Z. Jie and D. Gao, Tetrahedron Lett., 2017, 58, 1576-1578.

3 B. Marciniec, Hydrosilylation: a comprehensive review on recent advances, Springer Science \& Business Media, 2008.

4 R. Hofmann, M. Vlatković and F. Wiesbrock, Polymers, 2017, 9, 534 .

5 X. Du and Z. Huang, ACS Catal., 2017, 7, 1227-1243.

6 M. Oestreich, Angew. Chem., Int. Ed., 2016, 55, 494-499.

7 Y. Nakajima and S. Shimada, RSC Adv., 2015, 5, 2060320616.

8 T. K. Mukhopadhyay, F. Marco, T. L. Groy and R. J. Trovitch, J. Am. Chem. Soc., 2014, 136, 882-885.

9 Z. Yan, G. Uo Caihong and W. Haishun, Prog. Chem., 2014, 26, 345-357.

10 F. Hoffmann, J. Wagler, U. Böhme and G. Roewer, J. Organomet. Chem., 2012, 705, 59-69.

11 J. Sun and L. Deng, ACS Catal., 2015, 6, 290-300.

12 H. Zai, Y. Zhao, S. Chen, L. Ge, C. Chen, Q. Chen and Y. Li, Nano Res., 2018, 1-9.

13 J. L. Speier, J. A. Webster and G. H. Barnes, J. Am. Chem. Soc., 1957, 79, 974-979.

14 L. N. Lewis, J. Stein, Y. Gao, R. E. Colborn and G. Hutchins, Platin. Met. Rev., 1997, 41, 66-75.

15 H. Xie, H. Yue, W. Zhang, W. Hu, X. Zhou, P. Prinsen and R. Luque, Catal. Commun., 2018, 104, 118-122.

16 A. K. Roy, Adv. Organomet. Chem., 2007, 55, 1-59.

17 S. R. Arepalli, B. Shewit and B. S Lori, J. Long Term Eff. Med. Implant., 2002, 12, 299.

18 F. Li and Y. Li, J. Mol. Catal. A Chem., 2016, 420, 254-263.

19 D. Troegel and J. Stohrer, Coord. Chem. Rev., 2011, 255, 14401459.

20 M. Pagliaro, R. Ciriminna, V. Pandarus and F. Béland, Eur. J. Org. Chem., 2013, 2013, 6227-6235.

21 H. Maciejewski, A. Wawrzyńczak, M. Dutkiewicz and R. Fiedorow, J. Mol. Catal. A: Chem., 2006, 257, 141-148.

22 A. Wawrzyńczak, M. Dutkiewicz, J. Guliński, H. Maciejewski, B. Marciniec and R. Fiedorow, Catal. Today, 2011, 169, 6974.

23 M. Walczak, K. Stefanowska, A. Franczyk, J. Walkowiak, A. Wawrzyńczak and B. Marciniec, J. Catal., 2018, 367, 1-6.

24 Y. Chen, S. Ji, W. Sun, W. Chen, J. Dong, J. Wen, J. Zhang, Z. Li, L. Zheng and C. Chen, J. Am. Chem. Soc., 2018, 140, 8b-3121b.

25 W. Hu, H. Xie, H. Yue, P. Prinsen and R. Luque, Catal. Commun., 2017, 97, 51-55.

26 H. Zai, Y. Zhao, S. Chen, R. Wang, L. Ge, C. Chen and Y. Li, RSC Adv., 2016, 6, 98520-98527.

27 W. T. Koo, J. S. Jang, S. J. Choi, H. J. Cho and I. D. Kim, ACS Appl. Mater. Interfaces, 2017, 9, 18069-18077. 
28 L. Jian-Rong, S. Julian and Z. Hong-Cai, Chem. Rev., 2012, 112, 869-932.

29 L. Ye, Y. Bing and L. Jin-Liang, Chem. Commun., 2014, 50, 9969-9972.

30 C. K. Brozek, B. Luca, S. Tomohiro, T. V. Clark, N. López and D. Mircea, Chemistry, 2014, 20, 6871-6874.

31 G. Férey, C. Mellot-Draznieks, C. Serre, F. Millange, J. Dutour, S. Surblé and I. Margiolaki, Science, 2005, 309, 2040-2042.

32 R. Ricco, L. Malfatti, M. Takahashi, A. J. Hill and P. Falcaro, J. Mater. Chem. A, 2013, 1, 13033-13045.

33 A. Corma, H. García and F. X. Llabrés I Xamena, Chem. Rev., 2010, 110, 4606-4655.

34 G. Felipe, F. Hiroyasu, L. Seungkyu and O. M. Yaghi, J. Am. Chem. Soc., 2014, 136, 5271.

35 L. T. L. Nguyen, K. K. A. Le, H. X. Truong and N. T. S. Phan, Catal. Sci. Technol., 2012, 2, 521-528.

36 P. Falcaro, R. Ricco, A. Yazdi, I. Imaz, S. Furukawa, D. Maspoch, R. Ameloot, J. D. Evans and C. J. Doonan, Coord. Chem. Rev., 2016, 307, 237-254.
37 L. Ning, S. Liao, H. Cui, L. Yu and X. Tong, ACS Sustain. Chem. Eng., 2017, 6, 135-142.

38 S. M. Cohen, Chem. Rev., 2012, 112, 970-1000.

39 D. Jiang, L. L. Keenan, A. D. Burrows and K. J. Edler, Chem. Commun., 2012, 48, 12053-12055.

40 W. Zhenqiang, K. K. Tanabe and S. M. Cohen, Inorg. Chem., 2009, 48, 296-306.

41 F. David, A. Sonia and P. Catherine, ChemInform, 2010, 40, 7502-7513.

42 K. K. Tanabe and S. M. Cohen, Inorg. Chem., 2010, 49, 6766. 43 F. Zhang, Y. Jin, Y. Fu, Y. Zhong, W. Zhu, A. A. Ibrahim and M. S. El-Shall, J. Mater. Chem. A, 2015, 3, 17008-17015.

44 S. Xu, W. Meng, F. Bo, X. Han, Z. Lan, H. Gu, H. Li and L. Hui, J. Catal., 2018, 363, 9-17.

45 X. Wang, H. Li and X. J. Hou, J. Phys. Chem. C, 2012, 116, 19814-19821.

46 R. Bandari and M. R. Buchmeiser, Catal. Sci. Technol., 2011, 2, 220-226.

47 L. Ji, C. Yang, Z. Lei and T. Ma, J. Organomet. Chem., 2011, 696, 1845-1849.

48 J. F. Harrod and A. J. Chalk, J. Am. Chem. Soc., 1965, 87, 1133. 